\title{
Low-Cost Embedded Oximeter
}

\author{
M. Laghrouche, S. Haddab, S. Lotmani, K. Mekdoud, S. Ameur \\ LAMPA Laboratory, Department of Electronics, Mouloud MAMMERI University, Po Box 17 RP 15000, \\ Tizi Ouzou, Algeria. \\ larouche_67@yahoo.fr, shaddab@yahoo.fr
}

\begin{abstract}
Nowadays, many medical devices have been developed for the purposes of diagnosing and treatment. Wearable sensors and systems have evolved to the point that they can be considered ready for clinical application. The use of wearable monitoring devices that allow continuous or intermittent monitoring of physiological signals is critical for the advancement of both the diagnosis as well as treatment of diseases. Patient vital sign monitoring within hospitals requires the use of noninvasive sensors that are hardwired to bedside monitors. This paper describes the initial bench testing of a wireless wearable pulse oximeter. Arterial oxygen saturation in the patient's blood signal was measured with an optical sensor, and then converted to digital data using a microcontroller system. The digital data were then sent to a receiver where it is in $433 \mathrm{MHz}$ FM/FSK transmitter. At the receiver, the digital data were reconverted to analog signal to be monitored and recorded on the PC.
\end{abstract}

Keywords: Oximeter, Wireless, Telemedicine, FSK, Microcontroller.

\section{INTRODUCTION}

1 N TODAY'S healthcare practice, physicians have a need to monitor more than one medical parameter for patients who are either hospitalized or leading their normal daily activities at home or at work but in need of constant medical care. Pulse oximeters are devices that measure blood oxygen saturation and heart rate of a patient [1-4]. This feature provides a proper surveillance of the oxygen saturation. A physiological parameter measurement allows the identification of critical situations of the circulatory and pulmonary systems.

Decreased oxygen saturation is usually related to a diminution of arterial perfusion due to heart and pulmonary failure [5]. In critical situations, pulse oximetry allows to evaluate the cardiac and pulmonary function. This tool is also used during anesthesia and in other procedures like bronchoscopy, endoscopy, cardiac catheterization, exercise testing and sleep studies.

Telemedicine has been proposed as a means of improving the efficiency of the healthcare system, reducing the cost and stress caused by hospital environment. A wireless transmission is becoming increasingly popular in healthcare and biomedical engineering [6-9]. Modern data transmission wireless technologies give new possibilities to the designers of mobile medical equipment [6]. The objective of the study is the development of a low-cost wearable pulse oximeter for recording and monitoring saturation of arterial oxygen and heart beat rate. The system consists of an optical sensor, transmitter and receiver. The transmitter system includes an instrumentation amplifier, filter, microcontroller and FM/FSK transmitter. The receiver system consists of a FM/FSK receiver and Personal Computer (PC). The heart rate and the $\mathrm{SpO}_{2}$ level were observed for different subjects who had been subjected to physical exercise for a short period of time.

\section{MATERIAL AND METHODS}

\section{A. Principle of oximetry}

A pulse oximeter measures and displays the pulse rate and the saturation of hemoglobin in arterial blood [5]. This saturation of hemoglobin is a measure of the average amount of oxygen bound to each hemoglobin molecule. The absorption of visible light by a hemoglobin solution varies with oxygenation. The chemical binding of the different types of hemoglobin species changes the physical properties of the hemoglobin as well. The oxygen chemically combined with hemoglobin inside the red blood cells makes up nearly all of the oxygen present in the blood. Each time the heart muscle contracts, blood is ejected from the ventricles and a pulse of pressure is transmitted through the circulatory system. This pressure pulse, when traveling through the vessels, causes vessel wall displacement which is measurable at various points. In order to detect pulsatile blood volume changes, the photoelectric method is used [3],[4]. Light is emitted by a LED and transmitted through the artery and the resistance of the photo resistor is determined by the amount of light reaching it. With each contraction of the heart, blood is forced to the extremities and the amount of blood in the finger increases (Fig.1). It alters the optical density with the result that the light transmission through the finger is reduced and the resistance of the photo resistor or photodiode increases accordingly.

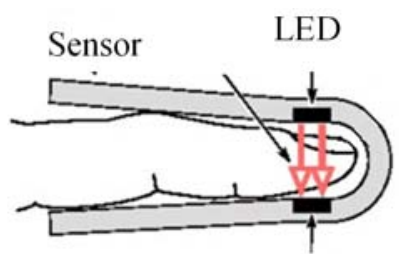

Photoresistor

Fig.1. Description of the optical sensor 


\section{B. System description}

Wireless pressure pulse system (Fig.2) includes a transmitter, receiver, and a PC which is used to record online data. The transmitter system includes an instrumentation amplifier and filter, a microcontroller and a FM/FSK transmitter. Pressure pulse signals of 3-6 mV amplitude and $0.05-150 \mathrm{~Hz}$ frequency components were measured with optical sensor from the finger by the signal perception circuit. At the transmitter, the signal was amplified, filtered, and converted to the 8-bit parallel digital data. This digital data were converted to the serial data by the 16F876 microcontroller at $4 \mathrm{MHz}$ operating frequency. Then a synchronization signal is added and 9600 baud data transmission speed is adjusted. The data are transmitted to space as electromagnetic waves by $433 \mathrm{MHz}$ FM/FSK transmitter [10] (FS1000A). The digital data from the transmitter are transmitted to the PC for record and monitoring.
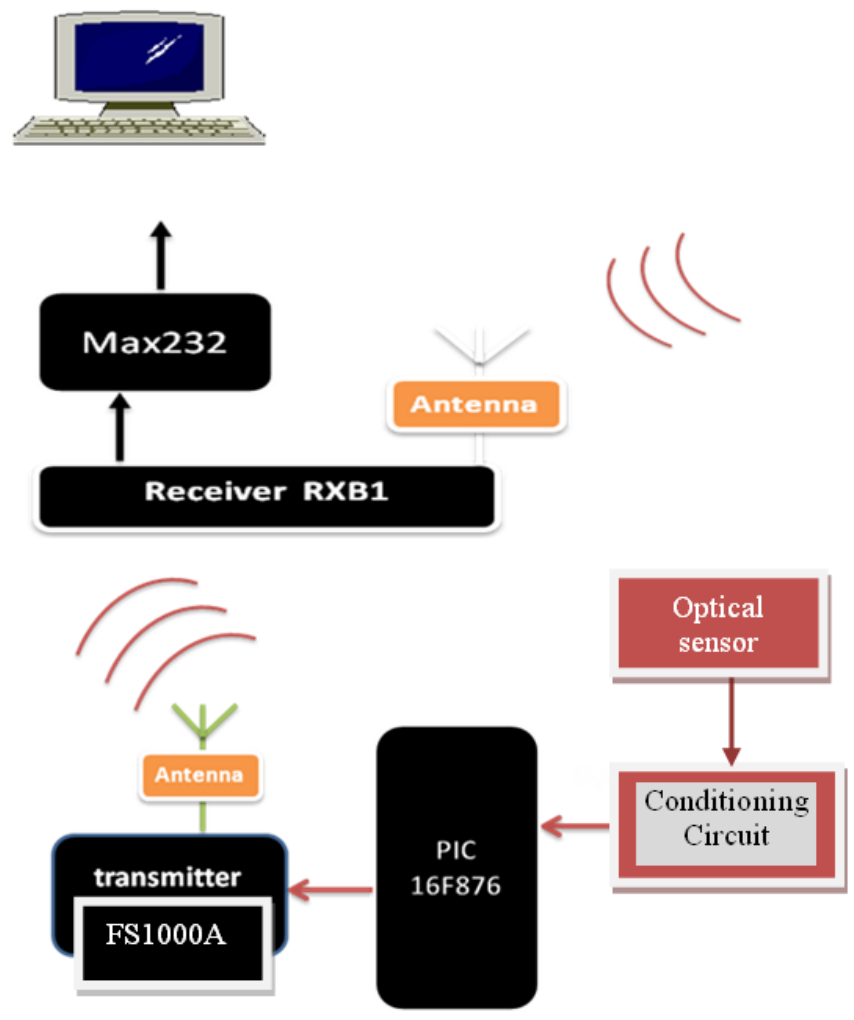

Fig.2. Block diagram of the data-acquisition system.

\section{Conditioning circuit}

The radiation amplifier consists of three blocks (Fig.3). The photo resistor is connected as a part of the voltage divider circuit and produces a voltage that varies with the amount of blood in the finger. The amplitude signal at the output of the sensor is around $6 \mathrm{mV}$ and it is amplified by the instrumentation amplifier which has $63 \mathrm{~dB}$ voltage gain and $110 \mathrm{~dB}$ common mode rejection ratio (CMRR). A filter was used for suppressing $50 \mathrm{~Hz}$ noise. Fig. 4 shows the signal after the conditioning circuit.

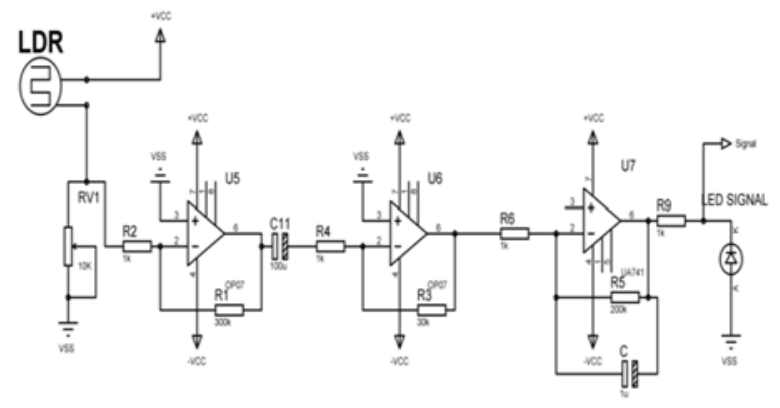

Fig.3. The conditioning circuit

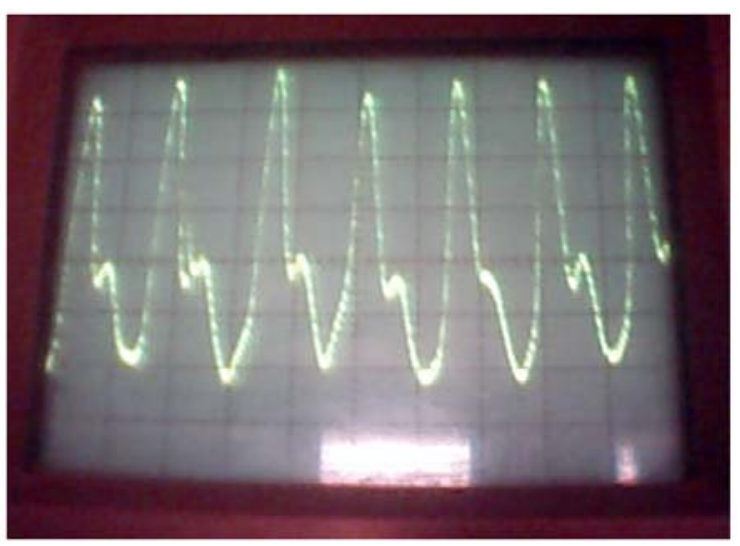

Fig.4. Oscilloscope view of optical sensor measurements after conditioning circuit

\section{Processing card}

The PIC 16F877 is an 8-bit microcontroller, which has an on-chip eight-channel 10-bit Analog-to-Digital Converter (ADC). The amplified and conditioned LDR signal is fed to channel-0 of the microcontroller. The microcontroller also continuously samples the optical signal on channel-0 with a sampling interval of 10 milliseconds. Samples are stored in EEPROM memory and transmitted directly to the serial port in asynchronous mode using the built-in UART of the PIC16F877, which is used to communicate with the high frequency transceiver using TTL voltage levels (Fig.5).The hardware for the current prototype oximeter is shown in Fig.6

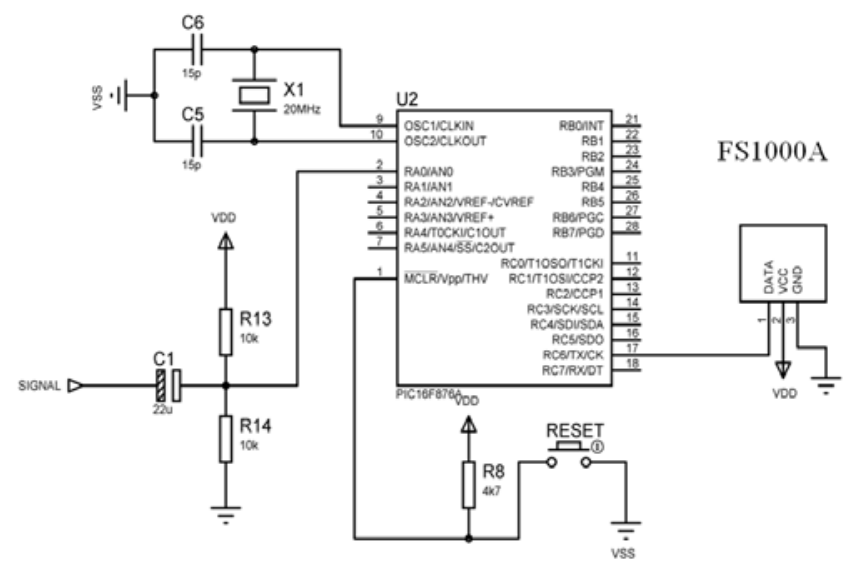

Fig.5. The processing card with high frequency transmission 


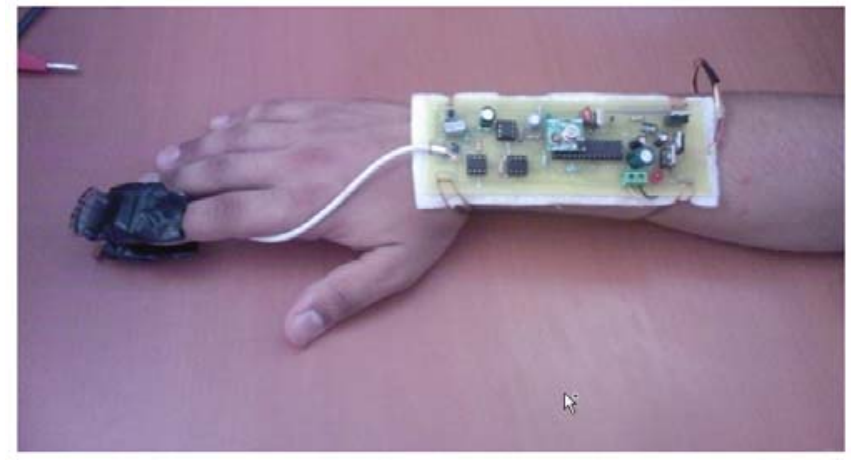

Fig.6. Hardware for the current prototype monitoring system

The Receiver system includes $433 \mathrm{MHz}$ FM/FSK receiver (Fig.7). Electromagnetic waves were caught by $433 \mathrm{MHz}$ FM/FSK receiver module. The digital data from the transmitter were transmitted to the PC for record and monitoring. The measurement results were recorded to the PC.
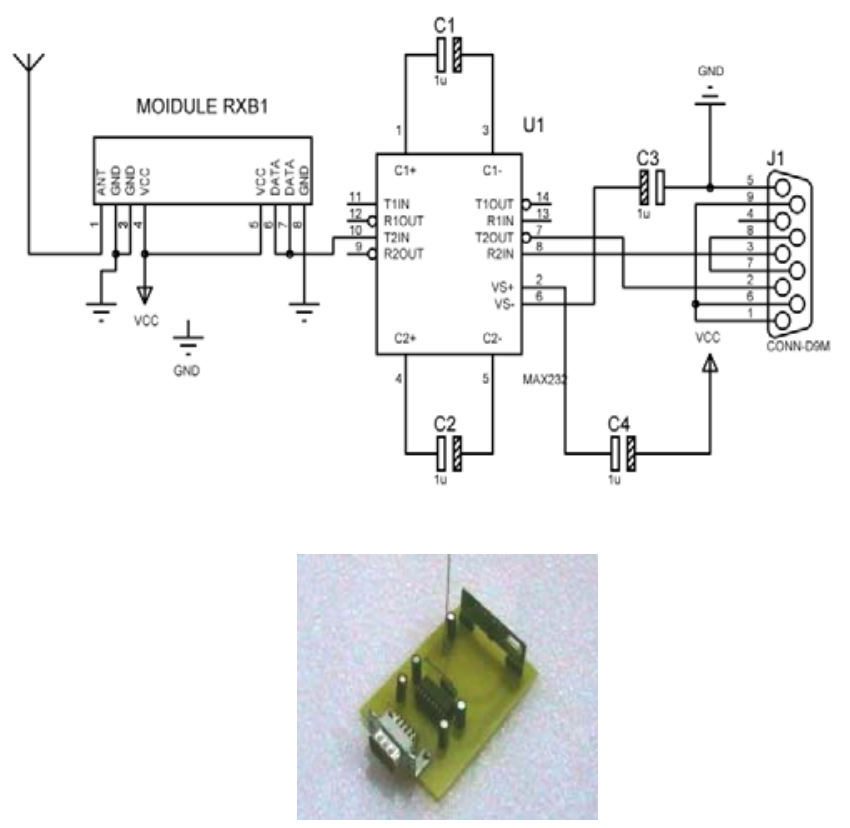

Fig.7. Receiver unit

\section{EXPERIMENTAL RESULTS}

For pulse oximetry measurements, several tests were performed on volunteers during normal breathing and forced breath-holding to see the repercussions on the pulse waveforms and, most importantly, on the oxygen saturation levels. Fig.8 shows the typical waveform produced when detecting the heart rate at the finger tip, for a normal person the frequency measured was $1.2 \mathrm{~Hz}$ which corresponds to 72 beats per minute. The peak-peak voltage is taken for each wavelength incident on the finger. For experimentation purposes the heart rate and the $\mathrm{SpO}_{2}$ level were observed after different subjects had been exercising for a short period of time.

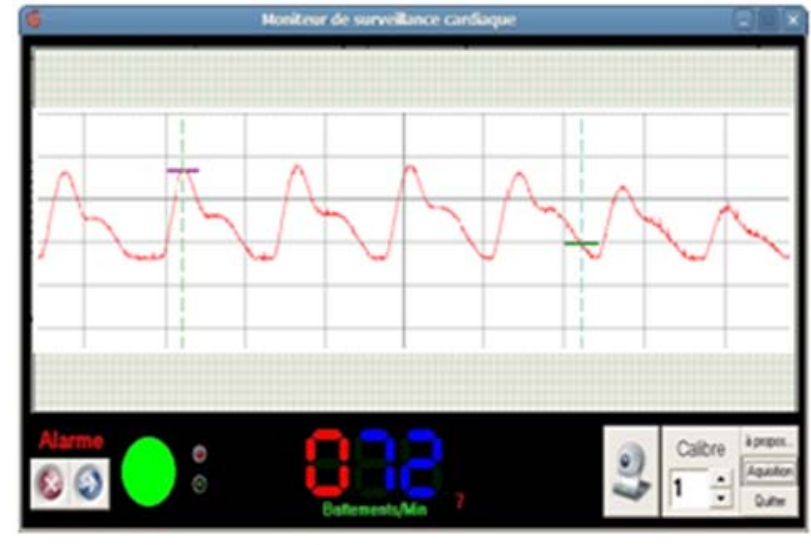

Fig.8. Typical wave form of the oximeter on the receiver unit

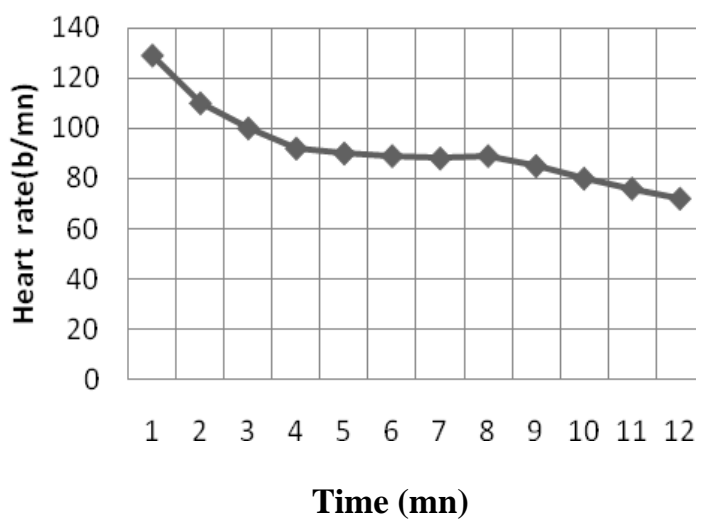

Fig.9. Heart rate observed after running.

Fig.9 shows how the heart rate gradually slowed down after the subject had completed a 12 minute run. For an athletic person, the heart rate is normally around 72 beats per minute at rest, so after a recovery time, the heart rate will return to this level. Fig.10 shows the oxygen saturation level of the same person after running. Overall, the $\mathrm{SpO}_{2}$ level was not affected greatly by running, mostly due to the fact that for an athletic person their oxygen level will not change much without physical exercise such as sprinting.

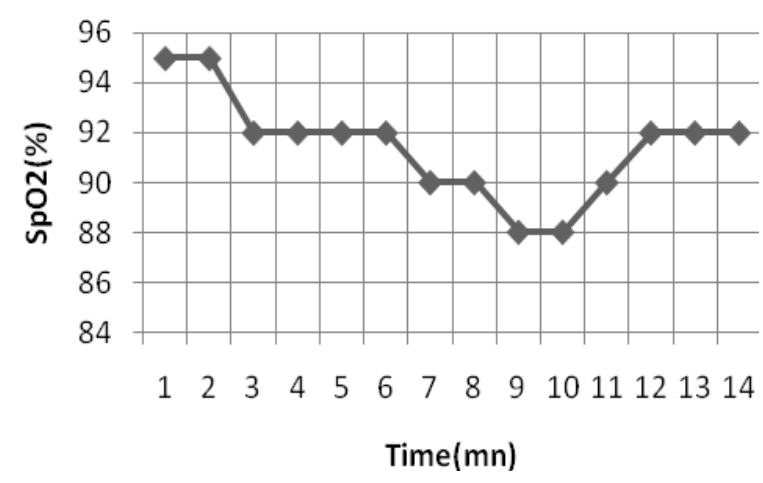

Fig.10. Oxygen level observed after running 


\section{CONCLUSION}

The design of a low-cost microcontroller based device for measuring the heart beat rate has been described. The device has the advantage that it can be used by non-professional people at home to measure the heart rate easily and safely. The components used in our designed device cost a few dollars. The main objectives of this work are to develop a medical sensor node for real-time measurement of arterial oxygen and heart beat rate monitoring using wireless transmission. The design of the pulse oximeter suggests the small size, light weight, standardized signal processing, and data processing and wireless communication capabilities. The application of WSN technology into elderly healthcare anticipates supporting the healthcare professionals effectively and economically in long-term care conveniences.

\section{REFERENCES}

[1] Goldman, J.M., Petterson, M.T., Kopotic, R.J., Barker, S.J. (2000). Signal extraction pulse oximetry. Journal of Clinical Monitoring and Computing, 16, 475-483.

[2] Polk, T., Walker, W., Hande, A., Bhatia, D. (2006). Wireless telemetry for oxygen saturation measurement. In BioCAS 2006 : Biomedical Circuits and Systems Conference. IEEE, 174-177.

[3] Johnston, W.S., Mendelson, Y. (2004). Extracting breathing rate information from a wearable reflectance pulse oximeter sensor. In Engineering in Medicine and Biology Society - IEMBS '04 : 26th Annual
International Conference of the IEEE. Vol. 2, 53885391.

[4] Mendelson, Y., Duckworth, R.J., Comtois, G. (2006). A wearable reflectance pulse oximeter for remote physiological monitoring. In Engineering in Medicine and Biology Society - EMBS '06 : 28th Annual International Conference of the IEEE, 912-915.

[5] Khandpur, R.S. (2003). Handbook of Biomedical Instrumentation. 2nd ed. New Delhi: Tata McGrawHill, 113.

[6] Tura, A., Badanai, M., Longo, O., Quareni, L. (2003). A medical wearable device with wireless Bluetooth based data transmission. Measurement Science Review, 3, 1-4.

[7] Paradiso, R, Loriga, G., Taccini, N. (2005). A wearable health care system based on knitted integrated sensors. IEEE Transactions on Information Technology in Biomedicine, 9, 337-344.

[8] Haddab, S., Laghrouche, M. (2009). Microcontrollerbased system for electrogastrography monitoring through wireless transmission. Measurement Science Review, 9 (5), 122-126.

[9] Asada, H. (2003). Mobile monitoring with wearable PPG sensors. IEEE Engineering in Medicine and Biology Magazine, 22, 28-39.

[10] Joshi, k., Rana, J. (2009). Wireless communication for tire pressure monitoring system based on ARM7TDMI. International Journal of Recent Trends in Engineering, 2 (8), 39-41. 